\title{
ON THE EXTENSION OF HOMEOMORPHISMS ON THE INTERIOR OF A TWO CELL
}

\author{
E. E. FLOYD
}

The subject under discussion in this paper is the study of the existence and properties of extensions of homeomorphisms of the interior $I$ of a two cell with boundary $C$ onto a plane bounded region. Particular emphasis will be placed on the action of the extension on $C$. Application of the topological results will then be made to conformal maps on the interior of the unit circle.

The hypothesis that $f(I)=R$ is a homeomorphism of the interior $I$ of a two cell with boundary $C$ onto a plane bounded region $R$ with boundary $F(R)$ will be assumed throughout the paper. The usual terminology of transformation theory will be used: the transformation $g(A)=B$ is said to be light if each $f^{-1}(x), x \in B$, is totally disconnected, and non-alternating if for each $x, y \in B, f^{-1}(x)$ does not separate $f^{-1}(y) .^{1}$

\section{Action of extensions on the boundary.}

THEOREM 1. Suppose $f$ is uniformly continuous. Then there exists a continuous extension $g$ of $f$ such that $g(\bar{I})=\bar{R}$ and $g=f$ on $I$. Moreover $g(C)=F(R)$ is a non-alternating transformation.

Proof. The existence of the extension is well known, since $f$ is uniformly continuous. Moreover $g(C)=F(R)$. To prove this, we notice that $g(\bar{I})$ is compact and must contain $\bar{R}$. Since $g(I)=R$, then $g(C) \supset F(R)$. Suppose $g(C) \neq F(R)$; then there is a point $x \in C$ such that $g(x) \in R$. Let $\left(x_{i}\right) \rightarrow x, x_{i} \in I$; then $\left(f\left(x_{i}\right)\right) \rightarrow g(x)$. Since $g(x) \in R$, then $\left(x_{i}\right) \rightarrow f^{-1} g(x) \in I$. This is a contradiction and $g(C)=F(R)$.

Suppose $g(C)=F(R)$ is not non-alternating; then there exist points $x_{1}, x_{2}, y_{1}, y_{2} \in C$ such that $g\left(x_{1}\right)=g\left(x_{2}\right), g\left(y_{1}\right)=g\left(y_{2}\right), g\left(x_{1}\right) \neq g\left(y_{1}\right)$, and $x_{1}+x_{2}$ separates $y_{1}+y_{2}$ on $C$. Let $A_{1}$ and $A_{2}$ be interiors of arcs $x_{1} x_{2}$ and $y_{1} y_{2}$ respectively, where $x_{1} x_{2} \subset I+x_{1}+x_{2}, y_{1} y_{2} \subset I+y_{1}+y_{2}, x_{1} x_{2} \cdot y_{1} y_{2}$ $=p=A_{1} A_{2}$. Both $g\left(x_{1} x_{2}\right)$ and $g\left(y_{1} y_{2}\right)$ are simple closed curves and $g\left(x_{1} x_{2}\right) \cdot g\left(y_{1} y_{2}\right)=f(p)$. Moreover points of $g\left(y_{1} y_{2}\right)$ are contained both in the interior and exterior of $g\left(x_{1} x_{2}\right)$. For $A_{1}$ separates $A_{2}$ into two parts, one in each component of $I-A_{1}$; then $f\left(A_{1}\right)$ separates $f\left(A_{2}\right)$ into two parts, one in each component of $R-f\left(A_{1}\right)$. But one compo-

Presented to the Society, April 27, 1946; received by the editors April 3, 1946.

${ }^{1}$ See G. T. Whyburn, Analytic topology, Amer. Math. Soc. Colloquium Publications, vol. 28, New York, 1942, pp. 127-129, 138-140, 165-170 for properties of nonalternating maps. 
nent of $R-f\left(A_{1}\right)$ is contained in the interior of $f\left(A_{1}\right)+g(x)$, the other in the exterior. This furnishes a contradiction, since $f(p)$ would then be a cut point of $g\left(y_{1} y_{2}\right)$, and the theorem is proved.

Definition. Let $R$ be a plane region with boundary $F(R)$; we say that $A$ is a cut into $R$ if $A \subset R$ and if there exists a point $x \in F(R)$ such that $A+x$ is an arc. We shall call $x$ the end of the cut $A$.

Definition. We say that $f$ possesses property $\mathrm{P}$ if

(1) $A$ is a cut into $R$ implies $f^{-1}(A)$ is a cut into $I$, and if

(2) $A_{1}$ and $A_{2}$ being cuts into $R$ with distinct ends implies $f^{-1}\left(A_{1}\right)$ and $f^{-1}\left(A_{2}\right)$ are cuts into $I$ with distinct ends.

LEMma 1. A necessary and sufficient condition that the boundary $F(R)$ of a bounded simply connected plane region $R$ be locally connected is that for each sequence $\left(x_{i}\right), x_{i} \in R$, such that $\left(x_{i}\right)$ converges to $x, x \in F(R)$, there exists an infinite subsequence $\left(y_{j}\right)$ of $\left(x_{i}\right)$ and an arc $a_{1} x, a_{1} x \subset R+x$, such that $a_{1} x \supset\left(y_{j}\right)$.

Proof. Let $R$ be a bounded simply connected plane region with locally connected boundary $F(R)$. To show the necessity portion of the lemma, we proceed to show two preliminary statements.

(1) There exists a sequence $\left(R_{i}\right)$ of regions contained in $R$ such that $R_{i+1} \subset R_{i}$, each $R_{i}$ possesses property $\mathrm{S},{ }^{2}$ each $R_{i}$ contains an infinite number of points of $\left(x_{i}\right)$, and the diameter of $R_{i}$ goes to 0 with $1 / i$.

To prove (1), we note first that $R$ possesses property $\mathrm{S}$, since $F(R)$ is locally connected..$^{3}$ Then $R$ may be decomposed into a finite number of regions, each with property $S$ and each of diameter less than $1 / 2.4$ One of these, say $R_{1}$, contains an infinite number of points of $\left(x_{i}\right)$ Then $R_{1}$ possesses property $\mathrm{S}$ and hence may be decomposed into a finite number of regions possessing property $\mathrm{S}$ and of diameter less than $1 / 4$. One of these, say $R_{2}$, contains an infinite number of points of $\left(x_{i}\right)$. By continuing the process, we obtain the required $\left(R_{i}\right)$.

(2) Let $A=p q-p$ be a cut into a region $R_{i}$ of (1), $p \in R \cdot F\left(R_{i}\right)$, $q \in R_{i}$. Let $R_{j} \subset R_{i}$ be such that $\bar{R}_{i} \cdot A=0$, and let $r \in R_{j}$. Then there exists an arc $q r, q r \subset\left(R_{i}-A\right)+q$, such that $q r \cdot F\left(R_{j}\right)$ consists of a single point, where $F\left(R_{n}\right)$ is used to denote the boundary of $R_{n}$.

To prove (2) we note first that $R_{i}-A$ is connected, since a cut into a region does not disconnect it. Furthermore $q$ is accessible from $R_{i}-A$ since a sufficiently small neighborhood of $q$ is contained in $R_{i}-A$ except for points of the arc $p q$. Let $q z$ be an arc contained in

${ }^{2}$ R. L. Moore, Concerning connectedness im kleinen and a related property, Fund. Math. vol. 3 (1922) pp. 232-237.

${ }^{3}$ R. L. Moore, loc. cit. p. 235.

4 G. T. Whyburn, loc. cit. p. 21. 
$\left(R_{i}-A\right)+q$, where $z \in R_{j}$. Let $y$ be the first point of $q z \cdot F\left(R_{j}\right)$ in the order $q$, z. Then $q y \subset\left(R_{i}-R_{i} \cdot \bar{R}_{j}\right)+y, y \in F\left(R_{j}\right)$. Since $R_{j}$ possesses property $\mathrm{S}$, each boundary point is accessible from $R .^{5}$ Then let $y r$ be an arc, $y r \subset R_{j}+y$. Then $q r=q y+y r$ is the desired arc, and (2) is justified.

We now proceed to show the necessity portion of the lemma. Let $y_{1} \in R_{1} \cdot\left(x_{i}\right)$. Let $R_{2}^{\prime}$ be such that $R_{2}^{\prime}$ is a member of the sequence $\left(R_{i}\right)$ and $y_{1} \notin \bar{R}_{2}^{\prime}$. Such an $R_{2}^{\prime}$ exists since $R_{i}$ is arbitrarily close to $x \in F(R)$ for $i$ sufficiently large. Let $y_{2} \in R_{2}^{\prime} \cdot\left(x_{i}\right)$. Let $y_{1} y_{2}=A$ be an arc contained in $R$ and such that $A \cdot F\left(R_{2}^{\prime}\right)$ consists of a single point. Such an arc may be constructed by an argument similar to the one used in (2). Let $R_{3}^{\prime}$ be such that $R_{3}^{\prime}$ is a member of $\left(R_{i}\right)$ and $y_{1} y_{2} \cdot \bar{R}_{3}^{\prime}=0$; let $y_{3} \in R_{3}^{\prime} \cdot\left(x_{i}\right)$. Since $R_{2}^{\prime} \cdot y_{1} y_{2}$ is a cut into $R_{2}^{\prime}$ we may use (2) to obtain an arc $y_{2} y_{3}$ contained in $\left(R_{2}^{\prime}-R_{2}^{\prime} \cdot y_{1} y_{2}\right)+y_{2}$ and intersecting $F\left(R_{3}^{\prime}\right)$ in a single point. Let $R_{4}^{\prime}$ be a member of $\left(R_{i}\right)$ such that $\bar{R}_{4}^{\prime} \cdot\left(y_{1} y_{2}+y_{2} y_{3}\right)=0$, let $y_{4} \in R_{4}^{\prime} \cdot\left(x_{i}\right)$, and continue the process indefinitely. We thus obtain a sequence $\left(y_{i-1} y_{i}\right)$ of arcs, $y_{i-1} y_{i} \subset R, y_{i} \in\left(x_{i}\right)$, $y_{i-1} y_{i}$ intersects $y_{1} y_{2}+y_{2} y_{3}+\cdots+y_{i-2} y_{i-1}$ in the single point $y_{i-1}$, and $y_{i-1} y_{i}$ approaches $x$ as a limit. Let $A=\sum_{i=2}^{\infty} y_{i-1} y_{i}+x$. Then every point of $A$ except $y_{1}$ and $x$ are cut points. Moreover, $A$ is compact, since the arcs $y_{i-1} y_{i}$ approach $x$ as limit. Then $A$ is an arc satisfying all the conditions, and the necessity is shown.

Suppose for each sequence the condition of the lemma is satisfied and suppose $F(R)$ is not locally connected. Then there is a point $x \in F(R)$ such that $x$ is not regularly accessible from $R .^{6}$ That is, for some $e>0$ there exists a sequence $\left(x_{i}\right), x_{i} \in R$ and $\left(x_{i}\right) \rightarrow x$, such that $x_{i}$ may not be joined to $x$ by an arc of diameter less than $e$ in $R+x$. By hypothesis, there exists an arc $a_{1} x \subset R+x$ such that $a_{1} x$ contains an infinite subsequence of $\left(x_{i}\right)$. This arc may be supposed to be of diameter less than $e$. This is a contradiction and the theorem is proved.

LEMma 2. If $F(R)$ is locally connected and $f$ possesses property $\mathrm{P}$, then $f$ is uniformly continuous.

Proof. Suppose $f$ is not uniformly continuous. Then for some $e>0$ and each $d_{i}$ of a sequence $\left(d_{i}\right) \rightarrow 0$, there exist $x_{i}, y_{i} \in I$ such that $\rho\left(x_{i}, y_{i}\right)<d_{i}$ and $\rho\left(f\left(x_{i}\right), f\left(y_{i}\right)\right) \geqq e$. We may suppose that $\left(x_{i}\right) \rightarrow x$; then $\left(y_{i}\right) \rightarrow x$ and $x \in C$. We may also suppose that $\left(f\left(x_{i}\right)\right) \rightarrow z_{1}$ and $\left(f\left(y_{i}\right)\right) \rightarrow z_{2}$. Then $z_{1} \neq z_{2}$. Moreover $z_{1} \in F(R)$ and $z_{2} \in F(R)$. For suppose $z_{1} \in R$; then $\left(x_{i}\right) \rightarrow f^{-1}\left(z_{1}\right) \in I$. But this is impossible; thus $z_{1}, z_{2} \in F(R)$. By Lemma 1 there exist cuts $A_{1}$ and $A_{2}$ which con-

${ }^{5}$ G. T. Whyburn, loc. cit. p. 111.

${ }^{6}$ G. T. Whyburn, loc. cit. p. 112. 
tain infinite subsequences of $\left(f\left(x_{i}\right)\right)$ and $\left(f\left(y_{i}\right)\right)$ respectively and which have $z_{1}$ and $z_{2}$ as ends. By property $\mathrm{P}, f^{-1}\left(A_{1}\right)$ and $f^{-1}\left(A_{2}\right)$ are cuts into $I$ with different ends. Since each of them must have $x$ as an end, this is a contradiction and the lemma is established.

THEOREM 2. Let $F(R)$ be locally connected. Then a necessary and sufficient condition that $f$ have property $\mathrm{P}$ is that there exist an extension $g$ of $f$, where $g(\bar{I})=\bar{R}, g=f$ on $I$, and $g(C)=F(R)$ is light and nonalternating.

Proof. Suppose $f$ has property P. Then by Lemma 2 and Theorem $1, g$ exists and $g(C)=F(R)$ is non-alternating. Suppose $g(C)=F(R)$ is not light; let $x \in F(R)$ be such that $g^{-1}(x)$ is not totally disconnected. Let $M$ be a nondegenerate component of $g^{-1}(x)$. Since $I+C$ is a two cell, there exists a set $N C I$ which is homeomorphic to the graph of the equation $y=\sin 1 / x, 0<x \leqq 1$, and such that $\bar{N}=N+M$. Then $f(N)$ is a cut into $R$ with end $x$. Since $f^{-1} f(N)=N$ is not a cut into $I$, then $f$ does not possess property $\mathrm{P}$, and we have a contradiction. This shows the necessity of the theorem.

Suppose $f$ may be extended to $g$, where $g(\bar{I})=\bar{R}, g=f$ on $I$, and $g(C)=F(R)$ is light and non-alternating. Let $A$ be a cut into $R$ with end $x$. Suppose $A=a_{1} x-x$. Let $\left(x_{i}\right) \rightarrow x$, where $x_{i} \in A$ and where $x_{i}$ precedes $x_{i+1}$ on the arc $a_{1} x$ ordered from $a_{1}$ to $x$. We may also suppose that $\left(f^{-1}\left(x_{i}\right)\right)$ converges to a point $y$. Let $A_{i}=x_{i} x_{i+1}$ be the subarc of $A$ joining $x_{i}$ to $x_{i+1}$. Then $\lim \sup f^{-1}\left(A_{i}\right) \subset g^{-1}(x)$ by the continuity of $g$. Since $\lim \inf f^{-1}\left(A_{i}\right) \supset y$, then $\lim \inf f^{-1}\left(A_{i}\right) \neq 0$ and $\lim \sup f^{-1}\left(A_{i}\right)$ is connected. ${ }^{7}$ Let $B=f^{-1}(A)$. Then $\lim \sup f^{-1}\left(A_{i}\right)$ $=\bar{B}-B$. Then $\bar{B}-B$ is both connected and totally disconnected, and hence is a single point. Then $B=f^{-1}(A)$ is a cut into $I$.

Suppose $A_{1}$ and $A_{2}$ are cuts into $R$ with ends $x_{1}$ and $x_{2}$ respectively, $x_{1} \neq x_{2}$, while $f^{-1}\left(A_{1}\right)$ and $f^{-1}\left(A_{2}\right)$ are cuts into $I$, both with end $y$. Then $g\left(f^{-1}\left(A_{1}\right)\right)=A_{1}$ and $g\left(f^{-1}\left(A_{2}\right)\right)=A_{2}$ are cuts into $I$, both with end $g(y)$. This is a contradiction and the theorem is established.

\section{Application to conformal maps.}

THEOREM 3. Let I be the interior of the unit circle, and let $f$ be a one-toone conformal map. Then $f$ possesses property P. ${ }^{8}$

CoRollary 3.1. Let $f(I)=R$ be a one-to-one conformal map of the interior $I$ of the unit circle $C$ onto a bounded plane region $R$ with bound-

${ }^{7}$ G. T. Whyburn, loc. cit. p. 14. 85.

${ }^{8}$ For proof, see C. Carathéodory, Conformal representation, London, 1932, pp. 82- 
ary $F(R)$. Then a necessary and sufficient condition that $f$ be extensible to $g$ on $\bar{I}$ is that $F(R)$ be locally connected. In case $F(R)$ is locally connected, and if $g$ denotes the extension, then the mapping $g(C)=F(R)$ is light and non-alternating.

PRoof. The sufficiency follows from Theorem 3 and Theorem 2. If $f$ is extensible to $g$ on $\bar{I}$, then $g(C)=F(R)$ gives a continuous map of the locally connected continuum $C$ onto $F(R)$. Then $F(R)$ itself must be locally connected.

CoROLlaRY 3.2. Let $f(I)=R$ be a one-to-one conformal map of the interior $I$ of the unit circle onto a bounded plane region $R$ with boundary $F(R)$. Then a necessary and sufficient condition that $f$ be uniformly continuous is that $F(R)$ be locally connected.

Proof. If $f$ is uniformly continuous, then $f$ is extensible to $g$ on $\bar{I}$ and $F(R)$ is locally connected by 3.1 . If $F(R)$ is locally connected, then $f$ is extensible to $g$ on $\bar{I}$ by 3.1. Then $g$ is uniformly continuous and so is $f$.

Corollary 3.3. The Osgood-Carathéodory Theorem. Let $f(I)=R$ be a one-to-one conformal map of the interior I of the unit circle $C$ onto the interior $R$ of a simple closed curve $J$. Then $f$ may be extended to $g$ on $\bar{I}$ such that $g(\bar{I})=\bar{R}$ is a homeomorphism.

PROoF. The mapping $g(C)=J$ is light and non-alternating; the only light and non-alternating transformation of one simple closed curve onto another is a homeomorphism. ${ }^{9}$ Hence $g(\bar{I})=\bar{R}$ is a homeomorphism.

Note. Another example of a map with property $\mathrm{P}$ is the inverse of the relative distance transformation ${ }^{10}$ on a plane bounded region with property $\mathrm{S}$. The following topological theorem is usually proven by means of this transformation :11 if $B$ is any boundary curve, there exists a light and non-alternating transformation $g(C)=B$, where $C$ is a simple closed curve. If $B$ is restricted to a boundary curve which contains at least one simple closed curve, we may prove the theorem by means of conformal maps as follows. We may suppose that $B$ is the boundary of a bounded plane region $R$. Then there exists a 1-1 conformal map $f$ of the interior of the unit circle onto $R$, since $R$ is simply connected. Then $f$ is extensible to $g$ on $\bar{I}$ and $g(C)=B$ is light and non-alternating.

UNIVERSITY OF VIRGINIA

${ }^{9} \mathrm{G}$. T. Whyburn, loc. cit. p. 165.

${ }^{10} \mathrm{G}$. T. Whyburn, loc. cit. pp. 155-162.

11 G. T. Whyburn, loc. cit. p. 166. 